\section{Getting a fix on SMA}

The predominant therapeutic strategy for treating SMA has focused on using what's already there in the genome. Take the SMN2 gene and make it work better, the reasoning goes. But another tactic involves putting back what's missing. The SMN2 gene doesn't work all that well anyway. Why not re-introduce fully functioning copies of SMN1 instead?

That's the approach being pursued by Brian Kaspar and his colleagues at the Nationwide Children's Hospital in Columbus, Ohio. "We're putting back exactly the target that one needs," Kaspar says. Using an adeno-associated virus as a vector, Kaspar's group has successfully introduced extra copies of the sequence for the human SMN protein into mouse models of SMA. Not only did this extend the animals' lifespans from a matter of weeks to months and sometimes years, but it also restored motor function to near-normal levels (Nat. Biotechnol. 28, 271-274, 2010).

"Gene delivery studies have demonstrated some of the most remarkable survival spans," Kaspar says. Similar preclinical results with comparable gene therapy products have also been reported by scientists at Genzyme, a Massachusetts-based subsidiary of the French drug giant Sanofi, and at Généthon, a nonprofit organization funded by the French Muscular Dystrophy Association (J. Clin. Invest. 120, 1253-1264, 2010; Hum. Mol. Genet. 20, 681-693, 2011).

With an eye to the clinic, Kaspar and his colleagues have also administered their viral construct to newborns from two larger animal species: pigs and cynomulgus macaque monkeys. The researchers showed that both blood- and brain-targeted injections led to successful viral uptake, with the introduced gene being expressed throughout the central nervous systems of these young

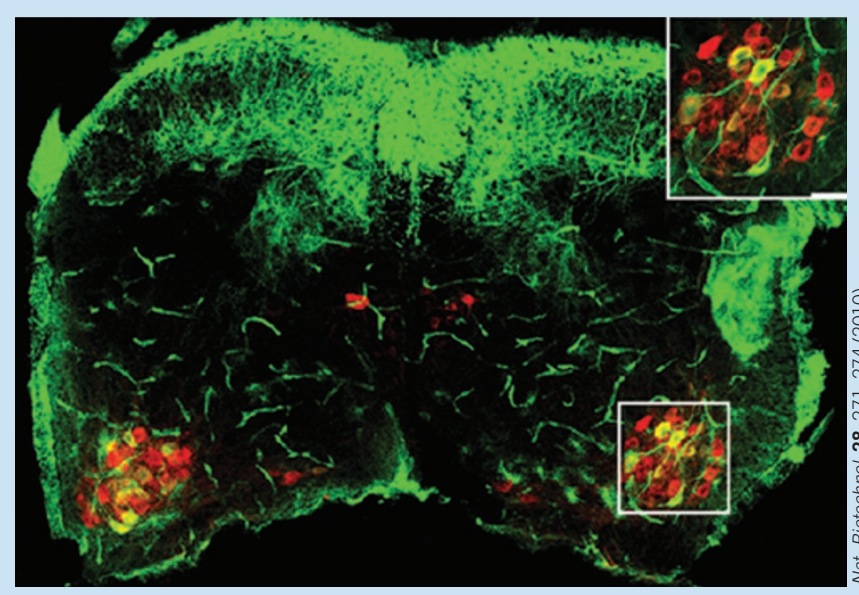

Deliver the goods: Injection of the Ohio group's gene therapy construct results in gene expression (green) within dorsal root ganglia and motor neurons (red) in the spinal cords of SMA mice.

animals with few adverse effects (Mol. Ther. 19, 1971-1980, 2011).

Now the Ohio team is working on generating the toxicology and pharmacokinetic data necessary for a full regulatory application. Led by Jerry Mendell, director of the Center for Gene Therapy at Nationwide Children's Hospital, the researchers intend to start clinical trials involving infants with type I SMA within the next year. "We think we have a very convincing argument," says Mendell, "and we'll have good safety data that we'll be able to move into a clinical trial in the zero- to six-month-old children." $-E D$ messenger RNA transcripts and primes them for destruction. By inhibiting that process, researchers have shown that they can increase the levels of SMN transcripts-both truncated and full length-resulting in elevated overall protein production and improvements in behavior and survival among mouse models of the disease ${ }^{4}$. "We're changing the steady-state metabolism of RNA," says James Rusche, head of research at Repligen.

In mouse studies, RG3039 increased the expression of SMN in central nervous system tissue by only about $50 \%$ - a "pretty modest" amount, admits Rusche. But that may be enough to generate real therapeutic benefits. "The production of SMN protein doesn't need to increase by much to go from almost no survival to almost no disease,"

Rusche says. "That appears to be true in the natural history of patients, and it appears to be true in the animal models as well."
Although Isis's drug was the first SMNtargeting agent tested in children, RG3039 was actually the first SMN-directed drug discovery program to enter human trials,

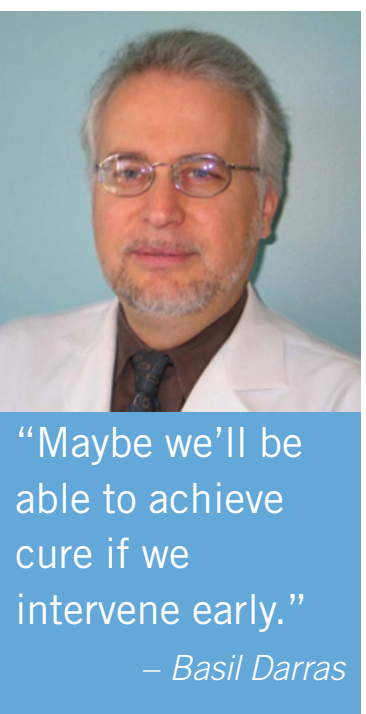
albeit in a healthy adult population. Repligen launched a single-dose, phase 1 trial involving 32 adult volunteers in May 2011. A year later, at the 2012 Annual Meeting of the American Academy of Neurology in New Orleans, the company announced that its oral liquid formulation of RG3039 proved safe, with molecular signs that the compound inhibited DcpS in peripheral blood cells. A multidose safety study among a second 32-person adult cohort kicked off in September of this year.

The drug's effect in peripheral blood cells is not inconsequential. Studies are beginning to show that SMA might not simply be a disease of the motor neurons, but one borne out in the peripheral muscles and other tissues, too. If validated, these emerging - and, it should be said, somewhat controversial-findings could mean that SMN-targeted drugs will need to work throughout the body, not just in the CNS, to have maximal therapeutic effect.

By default, that's already the case for small-molecule drugs such as the ones under development at Repligen and Roche. "If it gets in the brain, it gets in every cell in the body," notes Luca Santarelli, head of neuroscience at Roche, which is co-developing its splicingtargeted drug with PTC Therapeutics of Piscataway, New Jersey. It's trickier for oligonucleotide approaches, such as Isis's antisense therapy, which, in its first phase 1 study, was administered only into the CNS.

Although researchers found that delivering the Isis drug directly into the brain nearly doubled survival time in a mouse model of $\mathrm{SMA}^{3}$, systemic administration in the rodents-which usually die within two weeks-has been shown to extend median survival by up to eight months. "The results unequivocally say that you need high levels of SMN somewhere in peripheral tissues to have a healthy mouse," says study author Adrian Krainer, a molecular biologist at the Cold 